\title{
FROM DAMAGE MODELS TO SIR EPIDEMICS AND CASCADING FAILURES
}

\author{
MAUDE GATHY *** AND \\ CLAUDE LEFÈVRE, ${ }^{* * * *}$ Université Libre de Bruxelles
}

\begin{abstract}
This paper is concerned with a nonstationary Markovian chain of cascading damage that constitutes an iterated version of a classical damage model. The main problem under study is to determine the exact distribution of the total outcome of this process when the cascade of damages finally stops. Two different applications are discussed, namely the final size for a wide class of SIR (susceptible $\rightarrow$ infective $\rightarrow$ removed) epidemic models and the total number of failures for a system of components in reliability. The starting point of our analysis is the recent work of Lefèvre (2007) on a first-crossing problem for the cumulated partial sums of independent parametric distributions, possibly nonstationary but stable by convolution. A key mathematical tool is provided by a nonstandard family of remarkable polynomials, called the generalised Abel-Gontcharoff polynomials. Somewhat surprisingly, the approach followed will allow us to relax some model assumptions usually made in epidemic theory and reliability. To close, approximation by a branching process is also investigated to a certain extent.
\end{abstract}

Keywords: First-crossing problem; stability by convolution; damage model; generalised Abel-Gontcharoff polynomial; epidemic process; cascading failure; binomial, MarkovPólya and hypergeometric distributions; total outcome distribution; branching approximation

2000 Mathematics Subject Classification: Primary 60G40; 60E05; 62P05

\section{Introduction}

\subsection{Background}

In a recent paper, Lefèvre (2007) investigated a problem of first-crossing for the partial sums process of some nonstationary sequences. Let us briefly present the framework and the main result obtained.

Let $\left\{X_{t}, t=1,2, \ldots\right\}$ be a sequence of random variables taking on values in $\mathbb{N}=\{0,1$, $2, \ldots\}$. The basic assumption is that the variables $X_{t}$ are independent and of nonstationary distributions in the following specific sense. Firstly, the laws of all the $X_{t} \mathrm{~s}$ belong to a common parametric family of arithmetic laws, $\mathcal{L}(\theta)$ say, depending on a parameter $\theta \in \mathscr{D}=\mathbb{R}^{+}$or $\mathbb{N}$; let $p_{i}(\theta), i \geq 0$, be the associated probability mass function. Each $X_{t}$ has its own parameter value, and we write

$$
X_{1} \stackrel{\mathrm{D}}{=} \mathcal{L}\left(\theta_{0}+\theta_{1}\right) \quad \text { and } \quad X_{t} \stackrel{\mathrm{D}}{=} \mathcal{L}\left(\theta_{t}\right), \quad t \geq 2
$$

Received 14 August 2008; revision received 1 December 2008.

* Postal address: Université Libre de Bruxelles, Département de Mathématique, Campus de la Plaine CP 210, B-1050 Bruxelles, Belgium.

** Email address: maude.gathy@ulb.ac.be

*** Email address: clefevre@ulb.ac.be 
where $\stackrel{\text { D }}{=}$ ' denotes equality in distribution and $\theta_{0}+\theta_{1}, \theta_{2}, \theta_{3}, \ldots \in \mathcal{D}$. Secondly, the law $\mathcal{L}(\theta)$ is stable by convolution, which implies that, when $\theta \in \mathbb{R}^{+}, \mathcal{L}(\theta)$ is a compound Poisson law, and when $\theta \in \mathbb{N}, \mathcal{L}(\theta)$ is the $\theta$-fold convolution of $\mathcal{L}(1)$. As a direct consequence, for both cases, $\left\{e_{i}(\theta) \equiv p_{i}(\theta) / p_{0}(\theta), i \geq 0\right\}$ is a sequence of polynomials of degree $i$ in $\theta$ satisfying the following properties: $e_{0}(\theta)=1, e_{i}(0)=\delta_{i, 0}, i \geq 0$, and, for $\theta+\tilde{\theta} \in \mathcal{D}$,

$$
e_{i}(\theta+\tilde{\theta})=\sum_{j=0}^{i} e_{j}(\theta) e_{i-j}(\tilde{\theta}), \quad i \geq 0
$$

Owing to property (1.1), the polynomials $\left\{e_{i} / i !, i \geq 0\right\}$ are of binomial type (see Rota et al. (1973)) and the polynomials $\left\{e_{i}, i \geq 0\right\}$ are of convolution type (see Di Bucchianico (1997)).

Now, consider an associated partial sum process $\left\{m+S_{t}, t \geq 1\right\}$, where $m$ is any fixed integer greater than or equal to 1 and $S_{t}=X_{1}+\cdots+X_{t}, t \geq 1$. Since $\mathcal{L}(\theta)$ is stable by convolution, $S_{t} \stackrel{\mathrm{D}}{=} \mathcal{L}\left(\theta_{0}+\theta_{t}^{+}\right)$with $\theta_{t}^{+} \equiv \theta_{1}+\cdots+\theta_{t}, t \geq 1$. Let $T$ be the first-crossing time of the process $\left\{m+S_{t}, t \geq 1\right\}$ through the diagonal line of slope 1, i.e.

$$
T=\inf \left\{t \geq m: m+S_{t} \leq t\right\} .
$$

Clearly, $T$ corresponds to a first-meeting time, that is, $m+S_{T}=T$. The question under study is how to determine the distribution of the first-crossing level, $S_{T}$.

A key result established in Lefèvre (2007) (see formulae (4.5) and (4.7) in this paper) is that, at time $T$,

$$
\mathrm{P}\left(S_{T}=s\right)=p_{0}\left(\theta_{0}+\theta_{m+s}^{+}\right) \bar{G}_{s}\left(\theta_{0} \mid\left\{-\theta_{m+i}^{+}, i \geq 0\right\}\right), \quad s \geq 0
$$

Here, given a sequence of reals, $U=\left\{u_{i} \equiv-\theta_{m+i}^{+}, i \geq 0\right\}$, the attached sequence $\left\{\bar{G}_{s}(\theta \mid U)\right.$, $s \geq 0\}$ represents the family of generalised Abel-Gontcharoff $(A G)$ polynomials of degree $s$ in $\theta$. That family of polynomials was initially introduced in Picard and Lefèvre (1996) under a somewhat more general form of pseudopolynomials. The reader can find a concise presentation of these polynomials in Lefèvre (2007, Section 2).

We recall that the $\bar{G}_{s}(\theta \mid U) \mathrm{s}$ are built from the previous basic polynomials $\left\{e_{i}, i \geq 0\right\}$ using the simple recursion

$$
\bar{G}_{s}(\theta \mid U)=e_{s}(\theta)-\sum_{j=0}^{s-1} e_{s-j}\left(u_{j}\right) \bar{G}_{j}(\theta \mid U), \quad s \geq 0 .
$$

These polynomials also possess several nice algebraic and operational properties. In the special affine case where $u_{i}=a+b i, i \geq 0$, they reduce to

$$
\bar{G}_{s}(\theta \mid\{a+b i, i \geq 0\})=\frac{\theta-a}{\theta-a-b s} e_{s}(\theta-a-b s), \quad s \geq 0 .
$$

The standard AG polynomials due to Gontcharoff (1937) correspond to the particular case when $e_{i}(\theta)=\theta^{i} / i$ !,$i \geq 0$; they are denoted by $G_{s}(\theta \mid U)$. In the affine situation, these polynomials become, by (1.5), the classical Abel polynomials. 


\subsection{Overview}

The present paper, which is a continuation of Lefèvre (2007), is firstly motivated by applications to the modelling of epidemic processes. A number of works in epidemic theory are devoted to the study, exact or asymptotic, of the transient and final behaviours of various epidemic models. Much on this is presented in the comprehensive books of Daley and Gani (1999) and Andersson and Britton (2000).

Our attention will be focused on infectious diseases which are of the SIR (susceptible $\rightarrow$ infected $\rightarrow$ removed) schema. A statistic of great interest here is the final size $W_{e}$ of the epidemic, i.e. the total number of new infected cases at the end of the infectious process. It is well established that the AG polynomials provide a useful tool for expressing the exact distribution of the variable $W_{e}$ for many SIR epidemic models. We refer the reader to, e.g. Lefèvre and Picard (1990), (2005), Ball et al. (1997), Ball and O'Neill (1999), Ball and Lyne (2001), and Picard and Lefèvre (1990), (1991), (2003).

A closely related application in reliability theory will also be examined. It is concerned with a cascading failure model in a closed system of components, such as an electric power transmission system. This model was studied in Dobson et al. (2005) and Lefèvre (2006) and falls into the context of exchangeable cascading failures discussed in Lindley and Singpurwalla (2002). A central statistic here is the total number of new failures in the system, $W_{r}$, when the cascading process finally stops.

Our main purpose is to determine the exact distribution of the final epidemic size, $W_{e}$, and the total number of failures, $W_{r}$, within a unified and more general framework. For this, a novel model of cascading damage will be introduced by means of some nonstationary decreasing Markovian chain which comes to an end at an appropriate stopping time. Rather interestingly, this model may be viewed as an iterated cascading version of a classical damage model introduced in Rao (1965). The distribution of the total damage will again be expressed using the generalised AG polynomials. It will be derived by starting with (1.3) obtained for the law of the first-crossing level, $S_{T}$. To close, we will also investigate an approximation of this distribution by the total progeny law in some branching process.

\subsection{Rao's damage model}

As a preliminary, let us briefly present Rao's damage model. This model describes certain random phenomena which may be only partially observable due to a destructive or omission process. For example, it could represent the number of eggs laid by an insect, the number of car accidents declared by a driver, or the number of accidents in an energy company (see Charnet and Gokhale (2004)).

Formally, let $Z$ denote the random variable under study, which is assumed to take on values in $\mathbb{N}$ with probability mass function $p_{i}, i \geq 0$. Owing to possible deteriorations, $Z$ is subdivided into two parts, a lost set $X$ and a complementary undamaged set $Y$, both valued in $\mathbb{N}$ :

$$
Z=X+Y
$$

The survival distribution of $Y$ is given by the conditional law, whenever it exists,

$$
p_{j \mid i}=\mathrm{P}(Y=j \mid Z=i), \quad j=0, \ldots, i .
$$

This model has been discussed mainly in order to characterise certain distributions. For the sequel, it is worth mentioning two well-known results on this topic. The basic theorem, due to Rao and Rubin (1964), states that if $p_{0}<1$ and if, whenever $p_{n}>0$, the survival distribution is 
binomial, $\mathcal{B}(n ; p), p$ being fixed in $(0,1)$, then a condition of partial independence defined as

$$
\mathrm{P}(Y=j)=\mathrm{P}(Y=j \mid Z=Y \text { (i.e. } Z \text { is undamaged) }), \quad j \geq 0,
$$

is satisfied if and only if $Z$ has a Poisson distribution. Condition (1.7) and the associated characterisation of the Poisson law are called the Rao-Rubin condition and characterisation, respectively.

An important extension is provided by the following theorem proved in Shanbhag (1977). If $p_{0}<1$ and if there exist reals $a_{i}$ and $b_{i}, i \in \mathbb{N}$, satisfying $a_{i}>0$ for every $i$ and $b_{0}, b_{1}>0$, such that, whenever $p_{i}>0$, the survival distribution is of the form

$$
p_{j \mid i}=\frac{a_{j} b_{i-j}}{c_{i}}, \quad j=0, \ldots, i,
$$

where $\left\{c_{i}, i \geq 0\right\}$ is the convolution of $\left\{a_{i}, i \geq 0\right\}$ and $\left\{b_{i}, i \geq 0\right\}$, then the Rao-Rubin condition, (1.7), is satisfied if and only if $Z$ has a power series distribution. Moreover, (1.7) is also equivalent to the condition that the variables $Y$ and $X$ are independent. A number of other characterisations of this type can be found in the literature.

\section{A nonstationary damage process}

Let us consider a system of elements, individuals or machines for example, which is of size $Y_{0}=n \in \mathbb{N}$ at time 0 . The size of the system is interpreted as a measure of the 'safety' of the whole system. It could represent, for example, the number of individuals in good health or the number of functioning machines.

Just earlier, i.e. at time $0^{-}$, a damage phenomenon caused the destruction of $m \geq 1$ units, and this accident has weakened the safety of the actual $Y_{0}$ units. Each lost (i.e. damaged) unit, initial or subsequent, will generate, one after the other, a cascade of damages in an independent way. Roughly speaking, some kind of domino effect is engaged. A first lost unit damages the initial $Y_{0}=n$ units, so that at time $t=1, Y_{0}$ is decomposed as the sum of two $\mathbb{N}$-valued random variables, a damaged part $X_{1}$ and an undamaged part $Y_{1}$ (with $Y_{0}=X_{1}+Y_{1}$ ). Then, a second lost unit (if any) damages the remaining quantity $Y_{1}$, so that at time $2, Y_{1}$ is subdivided similarly into two parts, $X_{2}$ damaged units and $Y_{2}$ undamaged units (with $Y_{1}=X_{2}+Y_{2}$ ). By iteration, at time $t \geq 1$, the $t$ th lost unit (if it exists) damages the remaining $Y_{t-1}$ units, which are decomposed as

$$
Y_{t-1}=X_{t}+Y_{t}, \quad t \geq 1
$$

Let us now make precise the deterioration effects of every damage phenomenon that will occur. For this, we introduce the parametric family of distributions $\mathcal{L}(\theta), \theta \in \mathscr{D}$, stable by convolution, which was presented in the introduction (with probability mass function $p_{i}(\theta), i \geq 0$ ). The successive undamaged parts are defined through time-dependent survival distributions of the type (1.6). Specifically, the survival law at time 1 , denoted $p_{j \mid i}^{(1)}=\mathrm{P}\left(Y_{1}=j \mid Y_{0}=i\right)$, is given by

$$
p_{j \mid i}^{(1)}=\frac{p_{j}\left(\theta_{0}-\theta_{1}\right) p_{i-j}\left(\theta_{1}\right)}{p_{i}\left(\theta_{0}\right)}, \quad j=0, \ldots, i,
$$

whenever $p_{i}\left(\theta_{0}\right)>0$. Here, $\theta_{0}$ and $\theta_{1}$ are any given parameters in $\mathscr{D}$ satisfying the constraint $\theta_{1}<\theta_{0}$. Clearly, (2.2) is a probability law by virtue of the stability by convolution of $\mathcal{L}(\theta)$. We note that (2.2) corresponds to a simple parametric case of the conditional law (1.8) considered 
in Shanbhag (1977). Then, the remaining undamaged part $Y_{1}$ escapes damages at time 2 with a survival law $p_{j \mid i}^{(2)}=\mathrm{P}\left(Y_{2}=j \mid Y_{1}=i\right)$ of the same form but with different parameters:

$$
p_{j \mid i}^{(2)}=\frac{p_{j}\left(\theta_{0}-\theta_{1}-\theta_{2}\right) p_{i-j}\left(\theta_{2}\right)}{p_{i}\left(\theta_{0}-\theta_{1}\right)}, \quad j=0, \ldots, i,
$$

provided that $p_{i}\left(\theta_{0}-\theta_{1}\right)>0$, for some parameter $\theta_{2} \in \mathscr{D}$ satisfying $\theta_{1}+\theta_{2}<\theta_{0}$. By iteration, the survival law at every time $t \geq 1, p_{j \mid i}^{(t)}=\mathrm{P}\left(Y_{t}=j \mid Y_{t-1}=i\right)$, is given by

$$
p_{j \mid i}^{(t)}=\frac{p_{j}\left(\theta_{0}-\theta_{t}^{+}\right) p_{i-j}\left(\theta_{t}\right)}{p_{i}\left(\theta_{0}-\theta_{t-1}^{+}\right)}, \quad j=0, \ldots, i,
$$

provided that $p_{i}\left(\theta_{0}-\theta_{t-1}^{+}\right)>0$, where $\theta_{t}^{+}=\theta_{1}+\cdots+\theta_{t}<\theta_{0}$, with $\theta_{0}^{+} \equiv 0$.

\subsection{The total damage distribution}

Let us follow the process of undamaged parts, $\left\{Y_{t}, t \geq 1\right\}$, provided that damage phenomena continue to occur in the course of time. This process is a nonstationary Markovian chain whose transitions are ruled by the survival distributions (2.3). For the moment, it is convenient to assume that $Y_{0}$ is not equal to a constant $n$, but corresponds to a random variable with given law $\mathcal{L}\left(\theta_{0}\right)$.

Lemma 2.1. If $Y_{0} \stackrel{\mathrm{D}}{=} \mathcal{L}\left(\theta_{0}\right)$ then, for all $t \geq 1$,

$$
Y_{t} \stackrel{\mathrm{D}}{=} \mathcal{L}\left(\theta_{0}-\theta_{t}^{+}\right), \quad X_{t} \stackrel{\mathrm{D}}{=} \mathcal{L}\left(\theta_{t}\right),
$$

and $Y_{t}$ and $X_{t}$ are independent random variables.

Proof. We proceed by recurrence. For $t=1$, we have, by construction,

$$
\mathrm{P}\left(Y_{1}=j\right)=\sum_{i=j}^{\infty} \mathrm{P}\left(Y_{0}=i\right) p_{j \mid i}^{(1)}, \quad j \geq 0 .
$$

By assumption, $\mathrm{P}\left(Y_{0}=i\right)=p_{i}\left(\theta_{0}\right), i \geq 0$, so that inserting $(2.2)$ for $p_{j \mid i}^{(1)}$ yields

$$
\mathrm{P}\left(Y_{1}=j\right)=p_{j}\left(\theta_{0}-\theta_{1}\right) \sum_{i=j}^{\infty} p_{i-j}\left(\theta_{1}\right)=p_{j}\left(\theta_{0}-\theta_{1}\right),
$$

i.e. $Y_{1} \stackrel{\text { D }}{=} \mathcal{L}\left(\theta_{0}-\theta_{1}\right)$. Similarly, we see that

$$
\mathrm{P}\left(X_{1}=j\right)=\mathrm{P}\left(Y_{1}=Y_{0}-j\right)=\sum_{i=j}^{\infty} \mathrm{P}\left(Y_{0}=i\right) p_{i-j \mid i}^{(1)}=p_{j}\left(\theta_{1}\right), \quad j \geq 0 .
$$

Independence between $Y_{1}$ and $X_{1}$ then follows from the stability by convolution of the family $\mathcal{L}(\theta)$. Now, for any time $t \geq 2$, (2.1) gives

$$
\mathrm{P}\left(Y_{t}=j\right)=\sum_{i=j}^{\infty} \mathrm{P}\left(Y_{t-1}=i\right) p_{j \mid i}^{(t)}, \quad j \geq 0 .
$$

By recurrence, $\mathrm{P}\left(Y_{t-1}=i\right)=p_{i}\left(\theta_{0}-\theta_{t-1}^{+}\right)$for all $i$. Thus, using the survival law (2.3), we find that $Y_{t} \stackrel{\mathrm{D}}{=} \mathcal{L}\left(\theta_{0}-\theta_{t}^{+}\right)$, as announced. The law of $X_{t}$ and the independence between $Y_{t}$ and $X_{t}$ are easily obtained. This completes the proof. 
Obviously, the process $\left\{Y_{t}, t \geq 1\right\}$ is stochastically decreasing. As damage is caused by any previously lost unit, the deterioration process will stop as soon as there are no more lost units able to generate such damage. Let $S_{t}=X_{1}+\cdots+X_{t}=n-Y_{t}, t \geq 1$, be the total number of lost new units until time $t$. So, $m+S_{t}$ represents the total number of lost units able to cause damage until time $t$. On the other hand, the number of lost units susceptible to generate damage until time $t$ is, by construction, equal to $t$. Thus, cascading damage will continue provided that some lost units can still cause damage, i.e. as long as $m+S_{t}>t$. It will terminate when there remain no lost units able to cause damage, i.e. at time $T=\inf \left\{t \geq m: m+S_{t}=t\right\}$. This stopping time is identical to that considered in (1.2).

Let us now return to the case where $Y_{0}$ is a constant $n \in \mathbb{N}$. The statistic of interest is the total number of new units lost at the end $T$ of the cascading damage process; it corresponds to the variable $S_{T}=n-Y_{T}$. Owing to the above result from Lefèvre (2007), (1.3), we will derive the probability mass function of $S_{T}$ in terms of generalised AG polynomials.

Proposition 2.1. If $Y_{0}=n$ and $X_{0}=m$, then

$$
\mathrm{P}\left(S_{T}=s\right)=\frac{p_{n-s}\left(\theta_{0}-\theta_{m+s}^{+}\right) p_{0}\left(\theta_{m+s}^{+}\right)}{p_{n}\left(\theta_{0}\right)} \bar{G}_{s}\left(0 \mid\left\{-\theta_{m+i}^{+}, i \geq 0\right\}\right), \quad s=0, \ldots, n .
$$

Proof. A simple trick consists of supposing first that $Y_{0} \stackrel{\mathrm{D}}{=} \mathcal{L}\left(\theta_{0}\right)$. By Lemma 2.1 , the variables $X_{t}$ are then all independent and of laws $\mathcal{L}\left(\theta_{t}\right)$. Thus, the associated partial sums $S_{t}, t \geq 1$, are of laws $\mathcal{L}\left(\theta_{t}^{+}\right)$. Since the stopping time $T$ is defined by (1.2), the present problem is exactly the problem investigated in Lefèvre (2007), where here $\theta_{0}=0$. Consequently, (1.3) with $\theta_{0}=0$ provides the law of $S_{T}$, i.e.

$$
\mathrm{P}\left(S_{T}=s\right)=p_{0}\left(\theta_{m+s}^{+}\right) \bar{G}_{s}\left(0 \mid\left\{-\theta_{m+i}^{+}, i \geq 0\right\}\right), \quad n \geq 0 .
$$

Now, let us suppose that $Y_{0}=n$. We can write, for $s=0, \ldots, n$,

$$
\begin{aligned}
& \mathrm{P}\left(S_{T}=s \mid Y_{0}=n\right)=\frac{\mathrm{P}\left(S_{T}=s, Y_{0}=n, \text { and } Y_{0} \stackrel{\mathrm{D}}{=} \mathcal{L}\left(\theta_{0}\right)\right)}{\mathrm{P}\left(Y_{0}=n \text { and } Y_{0} \stackrel{\mathrm{D}}{=} \mathcal{L}\left(\theta_{0}\right)\right)} \\
& =\frac{\mathrm{P}\left(S_{T}=s \mid Y_{0} \stackrel{\mathrm{D}}{=} \mathcal{L}\left(\theta_{0}\right)\right) \mathrm{P}\left(Y_{0}=n \mid S_{T}=s, Y_{0} \stackrel{\mathrm{D}}{=} \mathcal{L}\left(\theta_{0}\right)\right)}{\mathrm{P}\left(Y_{0}=n \mid Y_{0} \stackrel{\mathrm{D}}{=} \mathcal{L}\left(\theta_{0}\right)\right)} .
\end{aligned}
$$

The probability in the denominator of (2.6) is $p_{n}\left(\theta_{0}\right)$ by definition. Now let us examine the two probabilities in the numerator. The former is obviously given by (2.5). In the latter, the event of concern means that $n-s$ units of the system escape all damage caused by the total number of lost units, $T$. Note that there are $m$ initial and $s$ new lost units, so that $T=m+s$. Thus, this event is equivalent to $Y_{m+s}=n-s$. As $Y_{0}$ is of law $\mathcal{L}\left(\theta_{0}\right)$ by the conditioning, Lemma 2.1 implies that $Y_{m+s} \stackrel{\mathrm{D}}{=} \mathcal{L}\left(\theta_{0}-\theta_{m+s}^{+}\right)$. So, the latter probability is equal to $p_{n-s}\left(\theta_{0}-\theta_{m+s}^{+}\right)$; hence, (2.4) is proved.

\subsection{The survival distributions}

Let us comment on the conditional survival law (2.3). We note that a parametric distribution of this type is discussed in Di Bucchianico (1997) in the context of umbral calculus.

Working with generating functions and using the stability by convolution of $\mathcal{L}(\theta)$, it is easily seen that law (2.3) is of mean

$$
\mathrm{E}\left(Y_{t} \mid Y_{t-1}=i\right)=i \frac{\theta_{0}-\theta_{t}^{+}}{\theta_{0}-\theta_{t-1}^{+}}, \quad i \geq 0
$$


independent of the specific law for $\mathcal{L}$. The other moments are not so simple. For the second factorial moment, a nice formula is

$$
\mathrm{E}\left(Y_{t}\left(Y_{t}-1\right) \mid Y_{t-1}=i\right)=i \frac{\theta_{0}-\theta_{t}^{+}}{\theta_{0}-\theta_{t-1}^{+}}\left(i-1-\sum_{j=1}^{i-1} \frac{f_{j}\left(\theta_{0}-\theta_{t}^{+}\right) f_{i-j}\left(\theta_{t}\right)}{f_{i}\left(\theta_{0}-\theta_{t-1}^{+}\right)}\right), \quad i \geq 0,
$$

where $\left\{f_{i}(\theta) \equiv i e_{i}(\theta) / \theta, i \geq 0\right\}$ forms a sequence of Sheffer polynomials of degree $i-1$ in $\theta$ (see, e.g. Picard and Lefèvre (1996)).

Let us now present a few particular survival distributions (2.3) obtained by choosing specific laws for $\mathcal{L}(\theta)$. When $\theta \in \mathbb{R}^{+}$, the following statements hold.

- If $\mathcal{L}(\theta)$ is a Poisson law, denoted by $\mathcal{P}(\theta)$, then, for $t \geq 1$ and $0 \leq j \leq i$,

$$
p_{j \mid i}^{(t)}=\left(\begin{array}{c}
i \\
j
\end{array}\right) \frac{\left(\theta_{0}-\theta_{t}^{+}\right)^{j} \theta_{t}^{i-j}}{\left(\theta_{0}-\theta_{t-1}^{+}\right)^{i}},
$$

which is a binomial law.

- If $\mathcal{L}(\theta)$ is a generalised Poisson law, denoted by $\mathcal{G} \mathcal{P}(\theta ; \beta)$ (i.e. $p_{i}(\theta)=\theta(\theta+\beta i)^{i-1}$ $\mathrm{e}^{-\theta-\beta i} / i$ !, $i \geq 0$, with $\left.\beta \in[0,1]\right)$, then, for $t \geq 1$ and $0 \leq j \leq i$,

$$
p_{j \mid i}^{(t)}=\left(\begin{array}{c}
i \\
j
\end{array}\right) \frac{\theta_{t}\left(\theta_{0}-\theta_{t}^{+}\right)}{\theta_{0}-\theta_{t-1}^{+}} \frac{\left(\theta_{0}-\theta_{t}^{+}+\beta j\right)^{j-1}\left(\theta_{t}+\beta(i-j)\right)^{i-j-1}}{\left(\theta_{0}-\theta_{t-1}^{+}+\beta i\right)^{i-1}},
$$

which is a quasibinomial II law (see, e.g. Johnson et al. (1992, pp. 102 and 396)).

- If $\mathcal{L}(\theta)$ is a negative binomial law, denoted by $\mathcal{N} \mathscr{B}(\theta ; p)$ (i.e. $p_{i}(\theta)=\left(\begin{array}{c}\theta+i-1 \\ i\end{array}\right)(1-p)^{i} p^{\theta}$, $i \geq 0$, with $p \in(0,1))$, then, for $t \geq 1$ and $0 \leq j \leq i$,

$$
\begin{aligned}
p_{j \mid i}^{(t)} & =\left(\begin{array}{c}
\theta_{0}-\theta_{t}^{+}+j-1 \\
j
\end{array}\right)\left(\begin{array}{c}
\theta_{t}+i-j-1 \\
i-j
\end{array}\right) /\left(\begin{array}{c}
\theta_{0}-\theta_{t-1}^{+}+i-1 \\
i
\end{array}\right) \\
& =\left(\begin{array}{c}
i \\
j
\end{array}\right) \frac{\left(\theta_{0}-\theta_{t}^{+}+j-1\right)_{[j]}\left(\theta_{t}+i-j-1\right)_{[i-j]}}{\left(\theta_{0}-\theta_{t-1}^{+}+i-1\right)_{[i]}}
\end{aligned}
$$

where $a_{[i]}=a(a-1) \cdots(a-i+1)$ with $a_{[0]}=1$, which is a particular Markov-Pólya law (also named the negative hypergeometric) (see, e.g. Johnson et al. (1992, p. 83)).

- If $\mathcal{L}(\theta)$ is a generalised negative binomial law, denoted by $\mathscr{g} \mathcal{N} \mathscr{B}(\theta ; \beta, p)$ (i.e. $p_{i}(\theta)=$ $[\theta /(\theta+\beta i)]\left(\begin{array}{c}\theta+\beta i \\ i\end{array}\right)(1-p)^{i} p^{\theta+\beta i-i}, i \geq 0$, with $p \in(0,1)$ and $\beta \in(1,1 /(1-p))$, then, for $t \geq 1$ and $0 \leq j \leq i$,

$$
\begin{aligned}
p_{j \mid i}^{(t)}= & \left(\begin{array}{c}
i \\
j
\end{array}\right) \frac{\theta_{t}\left(\theta_{0}-\theta_{t}^{+}\right)}{\theta_{0}-\theta_{t-1}^{+}} \frac{\theta_{0}-\theta_{t-1}^{+}+\beta i}{\left(\theta_{0}-\theta_{t}^{+}+\beta j\right)\left(\theta_{t}+\beta(i-j)\right)} \\
& \times \frac{\left(\theta_{0}-\theta_{t}^{+}+\beta j\right)_{[j]}\left(\theta_{t}+\beta(i-j)\right)_{[i-j]}}{\left(\theta_{0}-\theta_{t-1}^{+}+\beta i\right)_{[i]}},
\end{aligned}
$$

which is a particular generalised Markov-Pólya (or quasihypergeometric) law (see, e.g. Johnson et al. (1992, pp. 101 and 230)). 
When $\theta \in \mathbb{N}$, the following statement holds.

- If $\mathcal{L}(\theta)$ is a binomial law, denoted by $\mathcal{B}(\theta ; p)$ with $p \in(0,1)$, then, for $t \geq 1$ and $0 \leq j \leq i$,

$$
p_{j \mid i}^{(t)}=\left(\begin{array}{c}
\theta_{0}-\theta_{t}^{+} \\
j
\end{array}\right)\left(\begin{array}{c}
\theta_{t} \\
i-j
\end{array}\right) /\left(\begin{array}{c}
\theta_{0}-\theta_{t-1}^{+} \\
i
\end{array}\right),
$$

which is a hypergeometric law.

The survival binomial law (2.8), the Markov-Pólya law (2.9), and the hypergeometric law (2.11), will play special roles in the sequel. Note that the last two distributions do not depend on the parameter $p$ used in the corresponding laws $\mathcal{L}(\theta)$. Moreover, for these three laws, their factorial moments $\mathrm{E}\left(Y_{t}\left(Y_{t}-1\right) \mid Y_{t-1}=i\right)$ are explicitly given by

$$
\begin{gathered}
i(i-1) \frac{\left[\theta_{0}-\theta_{t}^{+}\right]^{2}}{\left[\theta_{0}-\theta_{t-1}^{+}\right]^{2}}, \quad i(i-1) \frac{\left[\theta_{0}-\theta_{t}^{+}\right]\left[\theta_{0}-\theta_{t}^{+}+1\right]}{\left[\theta_{0}-\theta_{t-1}^{+}\right]\left[\theta_{0}-\theta_{t-1}^{+}+1\right]}, \\
\text { and } i(i-1) \frac{\left[\theta_{0}-\theta_{t}^{+}\right]\left[\theta_{0}-\theta_{t}^{+}-1\right]}{\left[\theta_{0}-\theta_{t-1}^{+}\right]\left[\theta_{0}-\theta_{t-1}^{+}-1\right]},
\end{gathered}
$$

respectively. Thus, the variance of (2.8) is smaller or larger than the variance of (2.9) or, respectively, (2.11) when $\theta \in \mathbb{N}$. This suggests that the final influence of these three sampling methods might be comparable in some stochastic sense. The question remains open, however (see Figure 3 in Section 3).

\section{Application to SIR epidemics}

Consider a closed, homogeneous, and independently mixing population that is subjected to an epidemic of the SIR scheme. At the beginning it contains $n$ susceptibles, $m$ infectives, and no removed cases. Each infective, initial or future, acts independently and is able to infect any susceptible met during its infectious period. If ever contacted, a susceptible becomes an infective. At the end of the infectious period an infective recovers permanently from the disease and becomes a removed case without any possible influence on the spread of the disease. The epidemic ceases when there are no more infectives present, and this arises, almost surely, after a finite time $F$. The total number of new infected cases, $W_{e}$, is under consideration here.

Various models are proposed in the literature to represent SIR epidemic processes (see the books referred to in the introduction). The two most known SIR models are the so-called ReedFrost epidemic and general epidemic. They are examined below, as well as several nonstandard extensions of interest.

To find the distribution of $W_{e}$, we will operate a change of time scale in the model (as, e.g. in Lefèvre and Utev (1996)). First, the successive infectives are labelled $t=1,2, \ldots$, and a new time scale $t \in \mathbb{N}$ follows by considering the infectives, one after the other, during their infectious periods. Now, set $Y_{0}=n$ and, at each time $t \geq 1$, let us count the number of contacts, $X_{t}$, caused by the $t$ th infective among those individuals, in number $Y_{t-1}$, who escaped contacts with the previous $t-1$ infectives. By the subtraction $Y_{t-1}-X_{t}$, we then find the number $Y_{t}$ of individuals who are not yet infected at time $t$. This iterative procedure continues until time $T$ when there are no more infectives in the population, i.e. with $T$ given by (1.2). The final number of suseptibles is $Y_{T}=n-S_{T}$.

Obviously, the chosen time scale is quite different from the true time scale. The final outcome of the epidemic, however, is identical in both representations, i.e. $W_{e} \stackrel{\text { D }}{=} S_{T}$. Indeed, as far as 
$W_{e}$ is concerned, the real instant where an individual was infected is not relevant at all. In the new description, the epidemic model corresponds to a damage process such as described in Section 2.

It remains to specify how each infective contacts susceptibles during its infectious period, which will give the successive survival laws. Let us consider again a family of distributions $\mathcal{L}(\theta), \theta \in \mathscr{D}$, and assume that, for the $t$ th infective, if there are $i$ susceptibles present, the corresponding survival law $\left\{p_{j \mid i}^{(t)}, 0 \leq j \leq i\right\}$ is of the form (2.3). Then, by Proposition 2.1, the distribution of the final size $W_{e}$ is provided by (2.4).

A large variety of situations can be described by an appropriate choice of the survival law and the sequence of parameters $\theta_{t}$. Hereafter, we will first point out that the classical Reed-Frost epidemic and its variants are obtained when the survival distributions are chain binomial, and the parameters $\theta_{t}$ are geometrically decreasing. Then, we will focus on two new epidemic damage models that are built from the Markov-Pólya and hypergeometric survival laws.

\subsection{Reed-Frost epidemics}

3.1.1. The classical Reed-Frost model. For this model, we suppose that an infective goes through a latent period of length 1 , followed by an instantaneous infectious period. At that instant, the infective can contact any given susceptible with probability $p=1-q(0<q<1)$. So, if at time $t-1 \geq 0$ there are $I_{t-1}$ infectives, any of the $N_{t-1}$ susceptibles present will escape infectious contact at time $t$ with probability $q^{I_{t-1}}$. Thus, the conditional law of $N_{t}$ is of binomial type:

$$
N_{t} \mid\left(N_{t-1}, I_{t-1}\right) \stackrel{\mathrm{D}}{=} \mathcal{B}\left(N_{t-1}, q^{I_{t-1}}\right), \quad t \geq 1,
$$

where $N_{0}=n$ and $I_{0}=m$. At the end $F$ of the epidemic there will be a total number $W_{e}=n-N_{F}$ of newly infected cases.

Using the above equivalent representation, we obtain an associated damage process for which the individuals present escape contact with the $t$ th infective according to a binomial survival distribution:

$$
p_{j \mid i}^{(t)}=\left(\begin{array}{c}
i \\
j
\end{array}\right) q^{j}(1-q)^{i-j}, \quad j=0, \ldots, i .
$$

Note that law (3.2) is independent of $t$.

This situation can be viewed as a particular case of (2.3) when

$$
\mathcal{L}(\theta) \text { is Poisson } \mathcal{P}(\theta), \theta_{0}>0 \text {, and } \theta_{t}=\theta_{0} q^{t-1}(1-q), t \geq 1 .
$$

Then, indeed, $\theta_{t}^{+}=\theta_{0}\left(1-q^{t}\right), t \geq 1$, so that (2.8) reduces to (3.2).

Now, for the final size $W_{e},(2.4)$ combined with (3.3) yields

$$
\mathrm{P}\left(W_{e}=s\right)=n_{[s]} q^{(m+s)(n-s)} \theta_{0}^{-s} \bar{G}_{s}\left(0 \mid\left\{\theta_{0}\left(q^{m+i}-1\right), i \geq 0\right\}\right), \quad s=0, \ldots, n .
$$

For the law $\mathcal{P}(\theta)$, we have $e_{i}(\theta)=\theta^{i} / i !, i \geq 0$, which implies that the $\bar{G}_{s}$ reduce to the standard AG polynomials, $G_{s}$. Moreover, the following property then holds: given any reals $a$ and $b$,

$$
G_{s}(a+b \theta \mid a+b U)=b^{s} G_{s}(\theta \mid U), \quad s \geq 0 .
$$

Therefore, (3.4) simplifies to

$$
\mathrm{P}\left(W_{e}=s\right)=n_{[s]} q^{(m+s)(n-s)} G_{s}\left(1 \mid\left\{q^{m+i}, i \geq 0\right\}\right), \quad s=0, \ldots, n,
$$

a result already obtained in Lefèvre and Picard (1990) using a different method. 
3.1.2. A nonstationary Reed-Frost model. Let us assume that the number of individuals escaping contact with the $t$ th infective is still binomial but with a time-dependent parameter $0<q_{t}<1$, i.e.

$$
p_{j \mid i}^{(t)}=\left(\begin{array}{c}
i \\
j
\end{array}\right) q_{t}^{j}\left(1-q_{t}\right)^{i-j}, \quad j=0, \ldots, i .
$$

It is directly checked that in our modelling, (3.6) is obtained when assumptions (3.3) are modified to

$$
\mathcal{L}(\theta) \text { is Poisson } \mathcal{P}(\theta), \theta_{0}>0 \text {, and } \theta_{t}=\theta_{0} q_{1} \cdots q_{t-1}\left(1-q_{t}\right), t \geq 1,
$$

which yields $\theta_{t}^{+}=\theta_{0}\left(1-q_{1} \cdots q_{t}\right), t \geq 1$.

So, (3.5) is then generalised to

$$
\mathrm{P}\left(W_{e}=s\right)=n_{[s]}\left(q_{1} \cdots q_{m+s}\right)^{n-s} G_{s}\left(1 \mid\left\{q_{1} \cdots q_{m+i}, i \geq 0\right\}\right), \quad s=0, \ldots, n,
$$

which was also derived in Lefèvre and Picard (2005) by another argument.

Note that $q_{t}, t \geq 1$, represents the survival probability of any given susceptible who escaped contact with the $t-1$ first infectives. Thus, this is a conditional probability and it will generally be of the form $q_{t}=f_{t} / f_{t-1}$ with $f_{0}=1$. In this case, (3.7) is more compact since $q_{1} \cdots q_{m+i}=$ $f_{m+i}, i \geq 0$.

As an application, let us examine a Reed-Frost model with vaccination discussed in Lefèvre and Picard (2005). So, the classical model is considered with $q$ as the parameter of no contact, but now every susceptible is assumed to have received a vaccine that confers a random resistance to the disease. All the resistances are independent and have the same distribution, that of a variable $\varepsilon$ valued in $[0,1]$. Then, the modified model is of the previous nonstationary type, where $q_{t}=f_{t} / f_{t-1}$ with

$$
f_{t}=\mathrm{E}\left([q+(1-q) \varepsilon]^{t}\right), \quad t \geq 0 .
$$

In Figure 1 we consider three situations of this kind. The Reed-Frost plot gives the distribution function of $W_{e}$ when the resistance level $\varepsilon$ has a power-function distribution, i.e. $\mathrm{P}(\varepsilon \leq x)=x^{2}$ for $x \in[0,1]$. The upper bound plot corresponds to the approximation (usually named the leaky model) where $\varepsilon$ is replaced by its mean $\mathrm{E}(\varepsilon)=\frac{2}{3}$. The lower bound plot is the approximation (named all/nothing model) where $\varepsilon$ has a Bernoulli law of parameter $\mathrm{E}(\varepsilon)=\frac{2}{3}$. As proved in Lefèvre and Picard (2005), these two approximations provide upper and lower bounds for any distribution of $\varepsilon$ with given mean. Intuitively, it is natural that the safest case arises when the resistance level $\varepsilon$ is fixed instead of being a variable.

3.1.3. The randomised Reed-Frost model. This model allows us to take random levels of infectiousness into account. Each $t$ th infective does not contact any given susceptible present with a probability $Q_{t}$ which is assumed to be a random variable (especially to account for the variability of the length of its infectious period). All the $Q_{t}$ are independent and have the same distribution, that of $Q$ say.

Clearly, this model can be viewed as a randomised version of the nonstationary Reed-Frost epidemic. So, taking the expectation in (3.7) yields

$$
\mathrm{P}\left(W_{e}=s\right)=n_{[s]} \mathrm{E}\left(\left(Q_{1} \cdots Q_{m+s}\right)^{n-s} G_{n}\left(1 \mid\left\{Q_{1} \cdots Q_{m+i}, i \geq 0\right\}\right)\right), \quad s=0, \ldots, n .
$$

This result seems to be rather intricate. In Lefèvre and Picard (2005), however, the polynomial $G_{n}$ is expanded by Taylor's formula, and applying a nice property of the AG polynomials, they 


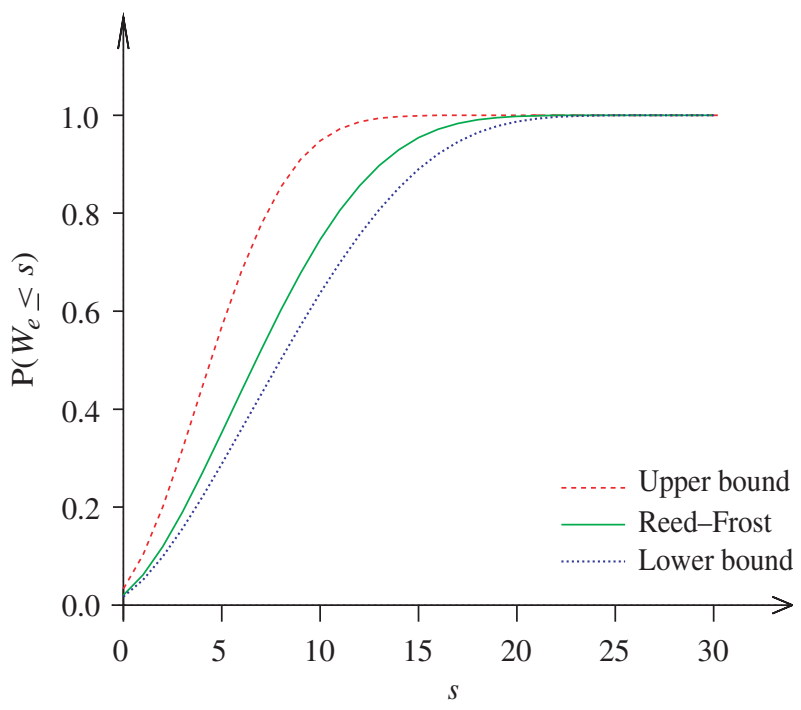

Figure 1: Graph of $\mathrm{P}\left(W_{e} \leq s\right), 0 \leq s \leq 30$, when $n=30, m=5$, and under (3.8), where $q=0.02$ and $\varepsilon=\frac{2}{3}$ almost surely (upper bound), $\mathrm{P}(\varepsilon \leq x)=x^{2}, x \in[0,1]$ (Reed-Frost), $\mathrm{P}(\varepsilon=1)=1-\mathrm{P}(\varepsilon=0)=\frac{2}{3}$ (lower bound).

deduced the following simplified formula:

$$
\mathrm{P}\left(W_{e}=s\right)=n_{[s]} \sum_{j=0}^{s} \frac{[q(n-j)]^{m+j}}{j !} G_{s-j}(0 \mid\{q(n-s+i), i \geq 0\}), \quad s=0, \ldots, n,
$$

where the expectation appears now only through the parameters $q(k)=\mathrm{E}\left(Q^{k}\right), k \geq 0$.

For instance, the general epidemic is a special case where the infectious periods are independent and exponentially distributed with parameter $\mu$, the generic variable being $D$ say, and pairs of individuals can contact according to independent Poisson processes of rate $\beta$. Then, $Q=\mathrm{e}^{-\beta D}$, which yields $q(k)=\mu /(\mu+k \beta), k \geq 0$.

\subsection{Markov-Pólya and hypergeometric epidemics}

In the Reed-Frost model, any given susceptible is contacted by an infective with a fixed probability $p=1-q$. Hereafter, we suppose that this probability depends on the number of contacts and the number of infections already made by the infective.

More precisely, in the previous epidemic scheme, consider time $t-1 \geq 0$ when there are $i$ susceptibles present, labelled $k=1, \ldots, i$. To follow the successive contacts made by the $t$ th infective among these $i$ susceptibles, we introduce the sequential probabilities:

$\mathrm{P}($ the $(k+1)$ th susceptible is infected at time $t \mid l$ among the first $k$ susceptibles were already infected $) \equiv p^{(t)}(k+1 \mid l)$

$$
=1-q^{(t)}(k+1 \mid l), \quad 0 \leq l \leq k \leq i .
$$

(i) As a first case, we make the assumption that, for any time $t \geq 1$,

$$
p^{(t)}(k+1 \mid l)=\frac{\theta_{t}+l}{\theta_{0}-\theta_{t-1}^{+}+k}, \quad l=0, \ldots, k .
$$


Thus, the probability to infect a first susceptible is equal to $\theta_{t} /\left(\theta_{0}-\theta_{t-1}^{+}\right)$. For the next susceptibles, the probability decreases with the number $k$ of previous contacts made, and it increases with the number $l$ of infection cases caused. In particular, the $(k+1)$ th susceptible has a more important risk than the first susceptible if the actual proportion of infections, $l / k$, is larger than the initial infection probability.

A situation like (3.10) might arise if, for instance, the infection power of the infective is not known with precision, but can be reevaluated after each contact by using a more adequate estimation. Note that, if the numbers $k$ and $l$ have no influence, (3.10) is replaced by $p^{(t)}=$ $\theta_{t} /\left(\theta_{0}-\theta_{t-1}^{+}\right)$and, as expected, $p^{(t)}$ reduces to $p=1-q$ in the Reed-Frost case.

Using (3.10), we then find that

$\mathrm{P}($ at $t$, the first $j$ susceptibles escape contact and the last $(i-j)$ susceptibles are contacted)

$$
\begin{aligned}
& =q^{(t)}(1 \mid 0) q^{(t)}(2 \mid 0) \cdots q^{(t)}(j \mid 0) p^{(t)}(j+1 \mid 0) p^{(t)}(j+2 \mid 1) \cdots p^{(t)}(i \mid i-j-1) \\
& =\frac{\left(\theta_{0}-\theta_{t}^{+}+j-1\right)_{[j]}\left(\theta_{t}+i-j-1\right)_{[i-j]}}{\left(\theta_{0}-\theta_{t-1}^{+}+i-1\right)_{[i]}}, \quad j=0, \ldots, i .
\end{aligned}
$$

Moreover, we see that, thanks to the expression in (3.10), the $\left(\begin{array}{l}j \\ i\end{array}\right)$ combinations of the $j$ surviving susceptibles all have the same probability. Therefore, $\left\{p_{j \mid i}^{(t)}, 0 \leq j \leq i\right\}$ is given by the MarkovPólya distribution (2.9). For this reason, the model will be named a Markov-Pólya epidemic. So here,

$$
\mathcal{L}(\theta) \text { is negative binomial, } \mathcal{N} \mathcal{B}(\theta ; p) .
$$

From (2.4) and (3.12), we obtain

$$
\begin{aligned}
\mathrm{P}\left(W_{e}=s\right)= & \frac{n_{[s]}}{(1-p)^{s}} \frac{\left(\theta_{0}-\theta_{m+s}^{+}\right) \cdots\left(\theta_{0}-\theta_{m+s}^{+}+n-s-1\right)}{\theta_{0} \cdots\left(\theta_{0}+n-1\right)} \\
& \times \bar{G}_{s}\left(0 \mid\left\{-\theta_{m+i}^{+}, i \geq 0\right\}\right), \quad s=0, \ldots, n .
\end{aligned}
$$

Now, let $\left\{\bar{G}_{s}^{a}(\theta \mid U), s \geq 0\right\}$ be the generalised AG polynomials associated to the sequence $\left\{a_{i}(\theta) \equiv e_{i}(\theta) /(1-p)^{i}, i \geq 0\right\}$. Applying Property A.1 in Appendix A, we deduce that (3.13) can be simplified to

$$
\begin{aligned}
\mathrm{P}\left(W_{e}=s\right)= & n_{[s]} \frac{\left(\theta_{0}-\theta_{m+s}^{+}\right) \cdots\left(\theta_{0}-\theta_{m+s}^{+}+n-s-1\right)}{\theta_{0} \cdots\left(\theta_{0}+n-1\right)} \\
& \times \bar{G}_{s}^{a}\left(0 \mid\left\{-\theta_{m+i}^{+}, i \geq 0\right\}\right), \quad s=0, \ldots, n .
\end{aligned}
$$

The parameter $p$ is no longer present here, which is not surprising since this was already the case in the survival law (2.9). Let us underline that the $\bar{G}_{s}^{a}$ are generalised, and not standard, AG polynomials.

Figure 2 provides a comparison between the classical Reed-Frost epidemic and the MarkovPólya epidemic built with the same geometric sequence of parameters $\left\{\theta_{t}, t \geq 0\right\}$. The sampling method used, of binomial or Markov-Pólya type, has a strong influence on the distribution of $W_{e}$. From (2.4), it is directly seen that $\mathrm{P}\left(W_{e}=0\right)$ is always smaller for the Reed-Frost model than for the Markov-Pólya model. Thus, a minor outbreak is more likely in the Markov-Pólya model. This is illustrated in Figure 2, which also shows that the two distribution functions finally cross each other. Thus, a major epidemic also appears to be more likely in the MarkovPólya epidemic. Intuitively, with this model, we may expect that, owing to assumption (3.10), a large number of infections is needed to generate some snowball effect in the contagion. 


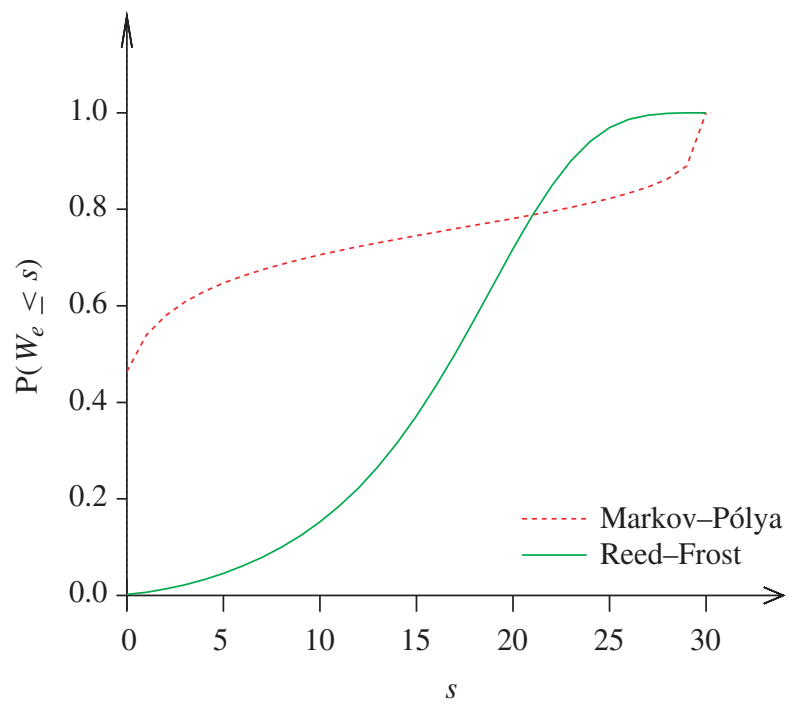

FiguRe 2: Graph of $\mathrm{P}\left(W_{e} \leq s\right), 0 \leq s \leq 30$, when $n=30, m=3$, and $\left\{\theta_{0}=1, \theta_{t}=q^{t-1}(1-q)\right.$, $t \geq 1\}$ with $q=0.96$ for the Markov-Pólya and classical Reed-Frost epidemics.

(ii) As a second case, let us assume that, for any time $t \geq 1$,

$$
p^{(t)}(k+1 \mid l)=\frac{\theta_{t}-l}{\theta_{0}-\theta_{t-1}^{+}-k}, \quad l=0, \ldots, k,
$$

provided that $l \leq \theta_{t}$ and $k<\theta_{0}-\theta_{t-1}$. This time, the probability to infect a susceptible increases with the number $k$ of previous contacts made, and it decreases with the number $l$ of infection cases caused. In particular, the infection risk for the $(k+1)$ th susceptible is now smaller than for the first susceptible if the actual proportion of infections, $l / k$, is larger than the initial risk $\theta_{t} /\left(\theta_{0}-\theta_{t-1}^{+}\right)$.

As a possible explanation for (3.14), we might think, for instance, that the infective carries germs, infectious or not, and some germs or infectious germs are destroyed after each contact made or, respectively, each infection caused.

Using (3.14) and following the same argument as in (3.11), we then find that $\left\{p_{j \mid i}^{(t)}, 0 \leq\right.$ $j \leq i\}$ is given by the hypergeometric distribution (2.11). For this reason, the model will be named a hypergeometric epidemic. So here,

$$
\mathcal{L}(\theta) \text { is binomial, } \mathcal{B}(\theta ; p) \text {. }
$$

From (2.4) and (3.15), we obtain

$$
\begin{aligned}
\mathrm{P}\left(W_{e}=s\right)= & \frac{n_{[s]}(1-p)^{s}}{p^{s}} \frac{\left(\theta_{0}-\theta_{m+s}^{+}\right) \cdots\left(\theta_{0}-\theta_{m+s}^{+}-n+s+1\right)}{\theta_{0} \cdots\left(\theta_{0}-n+1\right)} \\
& \times \bar{G}_{s}\left(0 \mid\left\{-\theta_{m+i}^{+}, i \geq 0\right\}\right), \quad s=0, \ldots, n .
\end{aligned}
$$

Let $\left\{\bar{G}_{s}^{a}(\theta \mid U), s \geq 0\right\}$ be the AG polynomials associated to the sequence $\left\{a_{i}(\theta) \equiv e_{i}(\theta)(1-\right.$ 


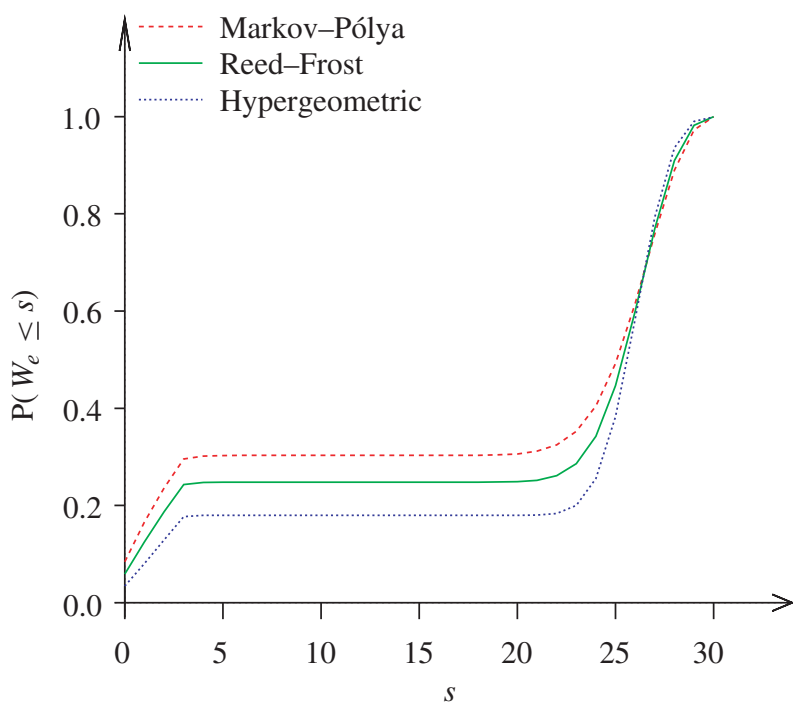

FiguRE 3: Graph of $\mathrm{P}\left(W_{e} \leq s\right), 0 \leq s \leq 30$, when $n=30, m=3$, and $\left\{\theta_{0}=100, \theta_{1}=\cdots=\right.$ $\left.\theta_{6}=3, \theta_{7}=\cdots=\theta_{13}=10, \theta_{14}=\cdots=0\right\}$ for the Markov-Pólya, nonstationary Reed-Frost and hypergeometric epidemics.

$\left.p)^{i} / p^{i}, i \geq 0\right\}$. By Property A.1, (3.16) then becomes

$$
\begin{aligned}
\mathrm{P}\left(W_{e}=s\right)= & n_{[s]} \frac{\left(\theta_{0}-\theta_{m+s}^{+}\right) \cdots\left(\theta_{0}-\theta_{m+s}^{+}-n+s+1\right)}{\theta_{0} \cdots\left(\theta_{0}-n+1\right)} \\
& \times \bar{G}_{s}^{a}\left(0 \mid\left\{-\theta_{m+i}^{+}, i \geq 0\right\}\right), \quad s=0, \ldots, n,
\end{aligned}
$$

the parameter $p$ being again absent as in the survival law (2.11).

In Figure 3 the hypergeometric epidemic is compared with the nonstationary Reed-Frost and Markov-Pólya epidemics for a sequence of parameters $\left\{\theta_{t}, t \geq 1\right\}$ valued in $\mathbb{N}$. From (2.4) we check that $\mathrm{P}\left(W_{e}=0\right)$ is always the smallest for the hypergeometric model. Thus, a minor outbreak is less likely for this epidemic. Furthermore, Figure 3 shows that the three distribution functions have a crossing point. So, a major epidemic also seems to be less likely in the hypergeometric model. By numerical calculation, we obtain $\mathrm{E}\left(W_{e}^{\mathrm{M}-\mathrm{P}}\right)=$ $18.9398, \mathrm{E}\left(W_{e}^{\mathrm{R}-\mathrm{F}}\right)=20.3166$, and $\mathrm{E}\left(W_{e}^{\mathrm{h}}\right)=21.9916$, using an obvious notation. From these observations, a plausible conjecture could be that $W_{e}^{\mathrm{M}-\mathrm{P}} \leq_{\mathrm{icv}} W_{e}^{\mathrm{R}-\mathrm{F}} \leq_{\mathrm{icv}} W_{e}^{\mathrm{h}}$, where ' $\leq_{\mathrm{icv}}$ ' denotes the increasing concave stochastic order (see, e.g. Lefèvre and Utev (1996)). Intuitively, it is not so surprising that, owing to assumption (3.14), the contagion in the hypergeometric model often becomes important but not extremely severe.

\section{Application to cascading failures}

A quite analogous damage model can be used in reliability to describe the occurrence of cascading failures in a closed system of $n$ interconnected similar components. This is not so surprising since in both applications, damage arises through some kind of domino effect amongst the individuals or components still present. 


\subsection{Binomial failures}

(i) Following Dobson et al. (2005) we suppose that in normal functioning conditions the $n$ components are able to bear loads $L_{1}, \ldots, L_{n}$, which are independent uniform $(0,1)$ random variables.

Step 0. For some technical reasons, an accident arises that implies an additional load $a$ to each component. As a consequence, any component $i$ will fail if its new load $L_{i}+a$ is greater than 1 , which happens with probability $\mathrm{P}\left(L_{i}>1-a\right)=a$; let $M_{0}$ be the number of failures.

Step 1. If there are new failures, a supplementary load $b$ per failed component is imposed to each functioning component. So, firstly a load $M_{0} b$ is added to the load of each of the $n-M_{0}$ remaining components. Amongst these, every component $i$ whose total load $L_{i}+a+b M_{0}$ is greater than 1 will fail; let $M_{1}$ be the number of failures.

Step 2. In an analogous way, a load $b M_{1}$ is then added to the $n-M_{0}-M_{1}$ remaining components, and the failure test is again applied. Such successive reload and damage processes will continue as long as possible.

Let $N_{t}, t \geq 1$, be the number of components functioning after $t$ steps. Then, $N_{t}=N_{t-1}-$ $M_{t-1}$, where $N_{0}=n$ and $M_{0} \stackrel{\mathrm{D}}{=} \mathscr{B}(n, a)$. This process is submitted to a cascading failure phenomenon of binomial type. More precisely, $N_{1} \stackrel{\mathrm{D}}{=} \mathscr{B}(n, 1-a)$, and at any time $t \geq 2$, every functioning component $i$ will remain active with the conditional probability

$$
\mathrm{P}\left(L_{i}+a+b\left(M_{0}+\cdots+M_{t-1}\right)<1 \mid L_{i}+a+b\left(M_{0}+\cdots+M_{t-2}\right)<1\right),
$$

which yields

$$
N_{t} \mid\left(N_{t-1}, N_{t-2}\right) \stackrel{\mathrm{D}}{=} \mathcal{B}\left(N_{t-1}, \frac{1-a-b\left(n-N_{t-1}\right)}{1-a-b\left(n-N_{t-2}\right)}\right), \quad t \geq 2 .
$$

The cascade stops at time $F$ when the loads of the remaining components are all smaller than 1 . The total number of failures is $W_{r}=n-N_{F}$. Note that in (4.1) it was implicitly assumed that $1-a-b(n-1)>0$. An analogous hypothesis is retained for the sequel. If this is not true (i.e. when $1-a-b i \leq 0$ for some $i \leq n-1$ ), the discussion can be easily adapted.

When interest is focused on $W_{r}$, we may operate a time scale change $t \in \mathbb{N}$ by considering the state of the system of components after the failure of any single component. In other words, the damage process of Section 2 is applicable here too. Denote by $X_{t}, t \geq 1$, the number of failures caused by the $t$ th failed component, and let $Y_{t}, t \geq 0$, be the number of components that are still functioning at time $t$, i.e. after the $t$ th failure. Then, $Y_{t}=Y_{t-1}-X_{t}, t \geq 1$, with $Y_{0}=n$. Because of the $t$ th failed component, if there were $i$ active components, the number of components that remain functioning is of survival law $\left\{p_{j \mid i}^{(t)}, 0 \leq j \leq i\right\}$ defined by (2.3). In the present case, this yields, for $t=1$,

$$
p_{j \mid n}^{(1)}=\left(\begin{array}{c}
n \\
j
\end{array}\right)(1-a)^{j} a^{n-j}, \quad j=0, \ldots, n,
$$

and, for $t \geq 2$,

$$
p_{j \mid i}^{(t)}=\left(\begin{array}{c}
i \\
j
\end{array}\right) \frac{(1-a-b(t-1))^{j} b^{i-j}}{(1-a-b(t-2))^{i}}, \quad j=0, \ldots, i .
$$

Comparing with (3.6), we observe that the model corresponds to a particular case of the nonstationary Reed-Frost model (2.3), with parameters

$$
q_{1}=1-a \quad \text { and } \quad q_{t}=\frac{1-a-b(t-1)}{1-a-b(t-2)}, \quad t \geq 2 .
$$


Note that

$$
f_{t}=q_{1} \cdots q_{t}=1-a-b(t-1), \quad t \geq 1,
$$

i.e. $f_{t}$ is a linear function of $t$.

The cascading failures stop at time $T$ when there are no more new failures. It is engaged by introducing one artificial infective, so that $T$ is represented by (1.2) with $m=1$. The total number of failures $W_{r}$ is distributed as $S_{T}=X_{1}+\cdots+X_{T}$. By Proposition 2.1, its law is provided by (3.7) with (4.2); hence,

$$
\mathrm{P}\left(W_{r}=s\right)=n_{[s]}(1-a-b s)^{n-s} G_{s}(1 \mid\{1-a-b i, i \geq 0\}), \quad s=0, \ldots, n .
$$

As the sequence $U$ defining $G_{s}$ in (4.3) is affine, we know by (1.5) that the $G_{s}$ reduce to the Abel polynomials. By substitution, (4.3) becomes

$$
\mathrm{P}\left(W_{r}=s\right)=\left(\begin{array}{l}
n \\
s
\end{array}\right) a(a+b s)^{s-1}(1-a-b s)^{n-s}, \quad s=0, \ldots, n,
$$

a result that can be found in Dobson et al. (1995).

(ii) In a recent paper, Lefèvre (2006) examined the same model, as well as several extensions, using a different formulation that has recourse to the order statistics of the loads $L_{1}, \ldots, L_{n}$. A connection with epidemics was also pointed out there, in somewhat intricate terms however.

So, let us now suppose that the initial loads $L_{i}$ are independent and identically distributed, but with an arbitrary distribution function $H$ on $(0,1)$, not necessarily uniform. Any component is still assumed to fail if its actual load is greater than 1 . In the present framework, we easily see that this variant is quite similar to the previous model, but with the parameters $q_{t}$ in (4.2) replaced by

$$
q_{1}=H(1-a) \quad \text { and } \quad q_{t}=\frac{H(1-a-b(t-1))}{H(1-a-b(t-2))}, \quad t \geq 2 .
$$

In particular, if the loads are of increasing exponential law, i.e. when $H(x)=\mathrm{e}^{x} /(\mathrm{e}-1)$ on $(0,1)$ say, then $q_{1}=\mathrm{e}^{1-a} /(\mathrm{e}-1)$ and $q_{t}=\mathrm{e}^{-b}, t \geq 2$. For an appropriate choice of $a$, all the $q_{t}$ are thus equal to each other, as in the classical Reed-Frost model (3.1).

More generally, for the additional loads in case of failures, we might now choose any fixed real sequence $w_{i}, i \geq 1$, not necessarily constant. Denote the cumulated weights by $v_{i}=w_{1}+\cdots+w_{i}, i \geq 1$, with $v_{n-1}<1$. Then, (4.5) is generalised as

$$
q_{1}=H(1-a) \quad \text { and } \quad q_{t}=\frac{H\left(1-a-v_{t-1}\right)}{H\left(1-a-v_{t-2}\right)}, \quad t \geq 2,
$$

where $v_{0}=0$. Finally, inserting (4.6) into (3.7) with $m=1$ yields

$$
\mathrm{P}\left(W_{r}=s\right)=n_{[s]}\left(H\left(1-a-v_{s}\right)\right)^{n-s} G_{s}\left(1 \mid\left\{H\left(1-a-v_{i}\right), i \geq 0\right\}\right), \quad s=0, \ldots, n,
$$

the $G_{s}$ again being standard AG polynomials.

As an illustration, suppose that, for security reasons, the system has to contain several redundant components. So, the first failures, in number $c \geq 0$ say, will have no influence on the functioning of the system and only the next failures will generate an additional load $b$ to the remaining components. In other words,

$$
v_{0}=v_{1}=\cdots=v_{c}=0 \quad \text { and } \quad v_{i}=b(i-c), \quad i \geq c+1 .
$$




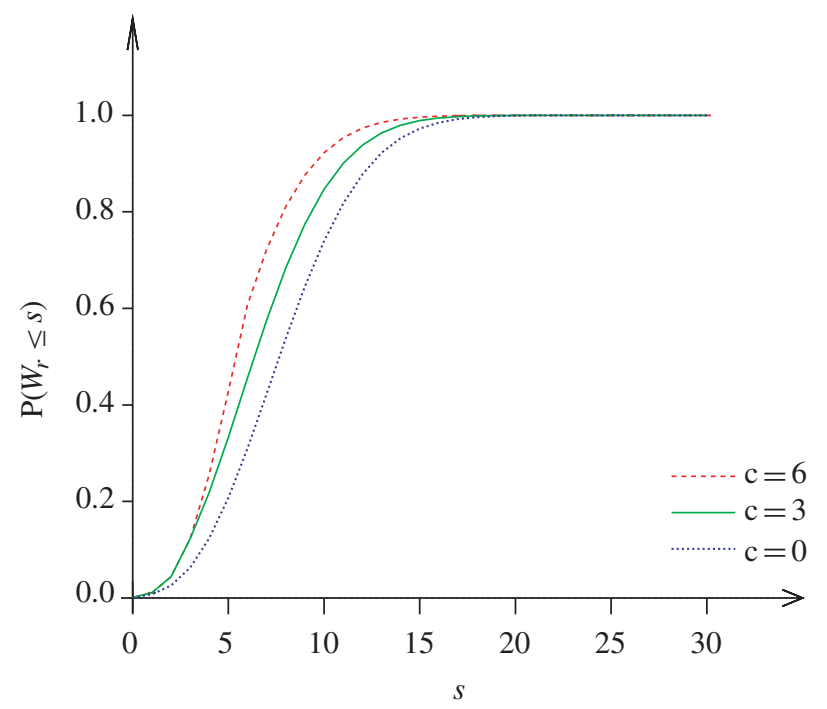

Figure 4: Graph of $\mathrm{P}\left(W_{r} \leq s\right), 0 \leq s \leq 30$, when $n=30, H$ uniform $(0,1), a=0.2, b=0.01$, and under (4.7) with $c=0,3$, and 6 .

Such a situation is considered in Figure 4, where the initial loads are uniform on $(0,1)$. Observe that, as expected, a higher security threshold $c$ implies a smaller total number of failures. The case in which $c=0$ gives the model without threshold examined in Dobson et al. (2005).

\subsection{Markov-Pólya and hypergeometric failures}

In the initial model (4.1), the probability for any given functioning component to fail due to the $t$ th failed component is equal to $a$ if $t=1$ and to $b /(1-a-b(t-2))$ if $t \geq 2$. This probability is now allowed to depend on the number of components tested and the number of failures already caused by the failed component.

Arguing as in (3.9), we consider time $t-1 \geq 0$ when there are $i$ components functioning, labelled $k=1, \ldots, i$, and we follow the successive possible failures generated by the $t$ th failed component. So, this time the probability

$$
p^{(t)}(k+1 \mid l)=1-q^{(t)}(k+1 \mid l), \quad 0 \leq l \leq k \leq i,
$$

represents the failure risk for the $(k+1)$ th component due to the $t$ th failed component, given that among the previous $k$ components, $l$ of them have already failed.

(i) Let us assume that, for any time $t \geq 1$,

$$
p^{(t)}(k+1 \mid l)=\frac{b+l}{a+b(n-t+1)+k}, \quad l=0, \ldots, k .
$$

Thus, the failure probability by a failed component decreases with the number $k$ of components tested and increases with the number $l$ of failures caused earlier.

Such a case might occur when, for instance, the resistance of the components can be reevaluated after each test by using a better calibrated estimation. Note that, if the numbers $k$ and $l$ have no influence, (4.8) is replaced by $p^{(t)}=b /(a+b(n-t+1))$, i.e. equivalently, 
$p^{(t)}=b /(a+b(n-1)-b(t-2))$; so, the form of $p^{(t)}$ looks like the one obtained for model (4.1).

Under (4.8), we then find that

$\mathrm{P}$ (at $t$, the first $j$ components still function and the last $(i-j)$ components have failed)

$$
=\frac{(a+b(n-t)+j-1)_{[j]}(b+i-j-1)_{[i-j]}}{(a+b(n-t+1)+i-1)_{[i]}}, \quad j=0, \ldots, i .
$$

Therefore, the survival law $\left\{p_{j \mid i}^{(t)}, 0 \leq j \leq i\right\}$ is given by the Markov-Pólya distribution (2.9), where $\theta_{0}=a+b n$ and $\theta_{t}=b$ for all $t \geq 1$.

Now, from (2.4), (3.12), and (4.9), we obtain

$$
\begin{aligned}
\mathrm{P}\left(W_{r}=s\right)= & \frac{n_{[s]}}{(1-p)^{s}} \frac{(a+b(n-s-1)) \cdots(a+b(n-s-1)+n-s-1)}{(a+b n) \cdots(a+b n+n-1)} \\
& \times \bar{G}_{s}(0 \mid\{-b(i+1), i \geq 0\}), \quad s=0, \ldots, n .
\end{aligned}
$$

As the family $U$ in $\bar{G}_{S}$ of (4.10) is affine, (1.5) is applicable, which yields

$$
\bar{G}_{s}(0 \mid\{-b(i+1), i \geq 0\})=\frac{e_{s}(b(s+1))}{s+1}, \quad s \geq 0 .
$$

Using (3.12) to express $e_{s}$, we then deduce that

$$
\begin{aligned}
\mathrm{P}\left(W_{r}=s\right)= & \left(\begin{array}{l}
n \\
s
\end{array}\right) \frac{(a+b(n-s-1)) \cdots(a+b(n-s-1)+n-s-1)}{(a+b n) \cdots(a+b n+n-1)} \\
& \times b[b(s+1)+1] \cdots[b(s+1)+s-1], \quad s=0, \ldots, n .
\end{aligned}
$$

(ii) We now make the assumption that, for any time $t \geq 1$,

$$
p^{(t)}(k+1 \mid l)=\frac{b-l}{a+b(n-t+1)-k}, \quad l=0, \ldots, k,
$$

provided that $l \leq b$ and $k<a+b(n-t+1)$. So, the failure probability now increases with the number $k$ of components tested and decreases with the number $l$ of failures caused. Such a situation might be plausible if, for instance, some security measures are relaxed with time but reinforced after the occurrence of failures.

Using (3.9), we find that the survival law $\left\{p_{j \mid i}^{(t)}, 0 \leq j \leq i\right\}$ corresponds to the hypergeometric distribution (2.11), where $\theta_{0}=a+b n$ and $\theta_{t}=b, t \geq 1$.

By adapting the argument followed for (4.11), we then obtain

$$
\begin{aligned}
\mathrm{P}\left(W_{r}=s\right)= & \left(\begin{array}{l}
n \\
s
\end{array}\right) \frac{(a+b(n-s-1)) \cdots(a+b(n-s-1)-n+s+1)}{(a+b n) \cdots(a+b n-n+1)} \\
& \times b(b(s+1)-1) \cdots(b(s+1)-s+1), \quad s=0, \ldots, n .
\end{aligned}
$$

Of course, a more general sequence $\left\{\theta_{t}, t \geq 1\right\}$ could be considered.

\section{Branching approximation}

It is well established in epidemic theory that in a large population the progress of the infectious disease for a minor epidemic can be approximated through a branching process (see, e.g. Ball 
(1983), Lefèvre and Picard (1990), Ball and Donnelly (1995), and Lefèvre and Utev (1999)). We investigate the validity of such an approximation for the total damage amount, $S_{T}$.

A central condition is that the number of elements in the system is quite large and the expected number of damages caused by each lost unit is roughly constant. In an epidemic context, this means that the population contains many susceptibles and any infective can only contact, on average, a limited number of individuals. Note that such an assumption is intuitive in order to get the announced result. Let us add an index $(n)$ in the notation to indicate a dependence on $n$; for instance, we write $\theta_{t} \equiv \theta_{t}^{(n)}, t \geq 0$. From (2.7), we see that the probability of the $t$ th lost unit causing damage to any present element is equal to $\theta_{t}^{(n)} /\left(\theta_{0}^{(n)}-\theta_{t-1}^{(n)+}\right)$. As $n \rightarrow \infty$, we are so led to assume that

$$
\frac{n \theta_{t}^{(n)}}{\theta_{0}^{(n)}} \rightarrow \theta_{l} \in \mathcal{D}, \quad t \geq 1 .
$$

A further condition is that the number of damaged cases at time 1, given a large initial system, converges in distribution to some random variable with a parametric law that is stable by convolution. Recall definition (2.2) for the probabilities $p_{n-j \mid n}^{(1)}$ of getting $j$ damages at time 1. Using condition (5.1), we thus assume that, as $n \rightarrow \infty$,

$$
\frac{p_{j}\left(\theta_{l} \theta_{0}^{(n)} / n\right) p_{n-j}\left(\theta_{0}^{(n)}-\theta_{l} \theta_{0}^{(n)} / n\right)}{p_{n}\left(\theta_{0}^{(n)}\right)} \rightarrow p_{l, j}\left(\theta_{l}\right), \quad j \geq 0,
$$

with $\quad \mathrm{P}_{l}\left(\theta_{l}\right) \equiv\left\{p_{l, j}\left(\theta_{l}\right), j \geq 0\right\}$ stable by convolution.

At first view, (5.2) might be satisfied if $\theta_{0}^{(n)} / n$ converges. We will see later that this is indeed true for the particular distributions discussed earlier. It is rather natural to suppose that $\theta_{0}^{(n)}$ is of order $n$, since $n$ represents a large fixed value of $Y_{0}^{(n)}$ and $Y_{0}^{(n)}$ is of law $\mathcal{L}\left(\theta_{0}^{(n)}\right)$.

Proposition 5.1. Under conditions (5.1) and (5.2), the total new damage, $S_{T}^{(n)}$, converges completely to the total new progeny in a Galton-Watson branching process with $m$ ancestors and offspring size distribution $\mathrm{P}_{l}\left(\theta_{l}\right)$.

Proof. Let us return to (2.4). From (5.1), the $\theta_{m+i}^{+(n)}$ can be approximated as

$$
\theta_{m+i}^{+(n)} \sim \frac{\theta_{l}(m+i) \theta_{0}^{(n)}}{n}, \quad i \geq 0,
$$

which constitutes a sequence of parameters affine in $i$. By (1.5) we then have

$$
\bar{G}_{s}\left(0 \mid\left\{-\theta_{m+i}^{+(n)}, i \geq 0\right\}\right) \sim \frac{m}{m+s} e_{s}\left(\frac{\theta_{l}(m+s) \theta_{0}^{(n)}}{n}\right), \quad s \geq 0 .
$$

Thus, inserting (5.3) into (2.4) yields

$$
\mathrm{P}\left(S_{T}^{(n)}=s\right) \sim \frac{m}{m+s} \frac{p_{s}\left(\theta_{l}(m+s) \theta_{0}^{(n)} / n\right) p_{n-s}\left(\theta_{0}^{(n)}-\theta_{l}(m+s) \theta_{0}^{(n)} / n\right)}{p_{n}\left(\theta_{0}^{(n)}\right)},
$$

and thanks to the first line of (5.2), we find that

$$
\lim _{n \rightarrow \infty} \mathrm{P}\left(S_{T}^{(n)}=s\right)=\frac{m}{m+s} p_{l, s}\left(\theta_{l}(m+s)\right), \quad s \geq 0 .
$$


Let $X_{l, t}\left(\theta_{l}\right), t \geq 1$, be a sequence of identically distributed random variables with law $\mathrm{P}_{l}\left(\theta_{l}\right)$. By the second line of (5.2) we can write that

$$
p_{l, s}\left(\theta_{l}(m+s)\right)=\mathrm{P}\left(\sum_{t=1}^{m+s} X_{l, t}\left(\theta_{l}\right)=s\right), \quad s \geq 0,
$$

so that (5.4) becomes

$$
\lim _{n \rightarrow \infty} \mathrm{P}\left(S_{T}^{(n)}=s\right)=\frac{m}{m+s} \mathrm{P}\left(\sum_{t=1}^{m+s} X_{l, t}\left(\theta_{l}\right)=s\right), \quad s \geq 0 .
$$

Now, the right-hand side of (5.5) is recognized to be the law of the total new progeny in the branching model indicated above (it suffices to use the classical ballot formula, given in, e.g. Takács (1989)). This completes the proof.

Note that setting $g(x), x \in[0,1]$, to be the probability generating function of $X_{l, 1}\left(\theta_{l}\right)$ (with law $\left.\mathrm{P}_{l}\left(\theta_{l}\right)\right),(5.5)$ is equivalent to

$$
\lim _{n \rightarrow \infty} \mathrm{P}\left(S_{T}^{(n)}=s\right)=\left.\frac{m}{m+s} \frac{1}{s !}\left\{\frac{\mathrm{d}^{s}}{\mathrm{~d} x^{s}}(g(x))^{m+s}\right\}\right|_{x=0}, \quad s \geq 0,
$$

i.e. the total damage $m+S_{T}^{(n)}$ converges completely to a delta Lagrangian distribution $L(m, g)$ (see, e.g. Consul and Famoye (2006)).

Corollary 5.1. Under (5.1) and (5.2), $S_{T}^{(n)}$ converges in distribution to the total new progeny in the above branching if and only if $\theta_{l} \leq 1$.

Proof. As complete convergence to a proper law is equivalent to convergence in distribution, we have to show that the total new progeny is finite almost surely if and only if $\theta_{l} \leq 1$. In fact, it can be proved that the Lagrangian law $L(m, g)$ in (5.6) is proper if and only if $\left.(\mathrm{d} g(x) / \mathrm{d} x)\right|_{x=1}=\mathrm{E}\left(X_{l, 1}\left(\theta_{l}\right)\right) \leq 1$ (see, e.g. Gathy (2007)). Now, using (2.7), we find that the expectation of the conditional law in the first line of (5.2) is equal to $\theta_{l}$ whatever the value of $n$. This implies that $\mathrm{E}\left(X_{l, 1}\left(\theta_{l}\right)\right)=\theta_{l}$; hence, the result.

By standard branching theory we then know that, if $\theta_{l}>1$, the total damage will be infinite with probability $1-\chi^{m}$, where $\chi$ is the positive root of the equation $g(x)=x$ in $[0,1]$.

Let us now consider the particular laws presented in Subsection 2.2.

Corollary 5.2. Under (5.1) and when

$$
\frac{\theta_{0}^{(n)}}{n} \rightarrow a \quad \text { with } a \theta_{l} \in \mathscr{D}
$$

- if $\mathcal{L}(\theta)=\mathscr{P}(\theta)$ then $\mathrm{P}_{l}\left(\theta_{l}\right)=\mathcal{P}\left(\theta_{l}\right)$;

- if $\mathcal{L}(\theta)=g \mathcal{P}(\theta ; \beta)$ then $\mathrm{P}_{l}\left(\theta_{l}\right)=\mathscr{g} \mathcal{P}\left(a \theta_{l} /(a+\beta) ; \beta /(a+\beta)\right)$;

- if $\mathcal{L}(\theta)=\mathcal{N} \mathscr{B}(\theta ; p)$ then $\mathrm{P}_{l}\left(\theta_{l}\right)=\mathcal{N} \mathscr{B}\left(a \theta_{l} ; a /(a+1)\right)$;

- if $\mathcal{L}(\theta)=g_{\mathcal{N}} \mathcal{B}(\theta ; \beta, p)$ then $\mathrm{P}_{l}\left(\theta_{l}\right)=g \mathcal{N} \mathcal{B}\left(a \theta_{l} ; \beta,(a+\beta-1) /(a+\beta)\right)$;

- if $\mathcal{L}(\theta)=\mathscr{B}(\theta ; p)$ then $\mathrm{P}_{l}\left(\theta_{l}\right)=\mathscr{B}\left(a \theta_{l} ; 1 / a\right)$. 
Proof. The derivation of $\mathrm{P}_{l}\left(\theta_{l}\right)$ is rather straightforward. In all situations, conditions (5.1) and (5.7) are found to guarantee the validity of condition (5.2). The case where $\mathcal{L}(\theta)$ is a generalised negative binomial law is discussed in Appendix A.

To close, we indicate that, as in epidemic theory, normal and Poisson approximations can also be established for the total damage, under appropriate conditions. This question is the object of a forthcoming work.

\section{Appendix A}

Let $\mathcal{L}(\theta) \equiv \mathcal{L}(\theta, c)$ be a generalised power series distribution with two parameters, i.e. of the form

$$
p_{i}(\theta, c)=\frac{a_{i}(\theta) c^{i}}{\eta(\theta, c)}, \quad i \geq 0,
$$

where $c>0$ is independent of $\theta, a_{i}(\theta) \geq 0$ for all $i \geq 0$, and $\eta(\theta, c)=\sum_{i=0}^{\infty} a_{i}(\theta) c^{i}$ (see, e.g. Johnson et al. (1992, p. 74)).

Property A.1. Suppose that $\left\{a_{i}(\theta), i \geq 0\right\}$ is a sequence of polynomials of convolution type, and let $\left\{\bar{G}_{s}^{a}(\theta \mid U), s \geq 0\right\}$ be the associated sequence of generalised AG polynomials. Define $e_{i}(\theta, c) \equiv p_{i}(\theta, c) / p_{0}(\theta, c)=a_{i}(\theta) c^{i}, i \geq 0$, and let $\left\{\bar{G}_{s}(\theta \mid U), s \geq 0\right\}$ be the associated sequence of generalised $A G$ polynomials. Then,

$$
\bar{G}_{s}(\theta \mid U)=c^{s} \bar{G}_{s}^{a}(\theta \mid U), \quad s \geq 0 .
$$

Proof. By (1.4), the $\bar{G}_{s}^{a}$ are built from the recursion

$$
\bar{G}_{s}^{a}(\theta \mid U)=a_{s}(\theta)-\sum_{j=0}^{s-1} a_{s-j}\left(u_{j}\right) \bar{G}_{j}^{a}(\theta \mid U), \quad s \geq 0,
$$

with $\bar{G}_{0}^{a}(\theta \mid U)=1$. Multiplying (A.2) by $c^{s}$ and using the definition of the $e_{i}(\theta, c)$, we see that the polynomials $c^{s} \bar{G}_{s}^{a}(\theta \mid U)$ satisfy (1.4); hence, identity (A.1) is proved.

In our context, this result is applicable to the negative binomial law $\mathcal{N} \mathscr{B}(\theta ; p)$, where $c=1-p$, and to the binomial law $\mathcal{B}(\theta ; p)$, where $c=p /(1-p)$.

The limit distribution $\mathrm{P}_{l}(\theta)$ in Corollary 5.2 is derived below when $\mathcal{L}(\theta)$ is a generalised negative binomial law. By (2.10), the conditional distribution $p_{n-j \mid n}^{(1)}$ considered in (5.2) then corresponds to a generalised Markov-Pólya law with

$$
\begin{aligned}
p_{n-j \mid n}^{(1)}= & \left(\begin{array}{c}
n \\
j
\end{array}\right) \frac{\left(\theta_{l} \theta_{0}^{(n)} / n\right)\left(\theta_{0}^{(n)}-\theta_{l} \theta_{0}^{(n)} / n\right)}{\theta_{0}^{(n)}} \frac{\theta_{0}^{(n)}+\beta n}{\left(\theta_{0}^{(n)}-\theta_{l} \theta_{0}^{(n)} / n+\beta(n-j)\right)\left(\theta_{l} \theta_{0}^{(n)} / n+\beta j\right)} \\
& \times\left(\frac{\theta_{l} \theta_{0}^{(n)}}{n}+\beta j\right)_{[j]} \frac{\left(\theta_{0}^{(n)}-\theta_{l} \theta_{0}^{(n)} / n+\beta(n-j)\right)_{[n-j]}}{\left(\theta_{0}^{(n)}+\beta n\right)_{[n]}}, \quad j=0, \ldots, n .
\end{aligned}
$$


Proof of Corollary 5.2 when $\mathcal{L}(\theta)=g_{\mathcal{N}} \mathscr{B}(\theta ; \beta, p)$. Under (5.1) and (5.7), direct approximations in the successive factors of (A.3) yield

$$
\begin{aligned}
p_{n-j \mid n}^{(1)} & \sim \frac{n^{j}}{j !} a \theta_{l} \frac{1}{a \theta_{l}+\beta j}\left(a \theta_{l}+\beta j\right)_{[j]} \frac{\left(a n-a \theta_{l}+\beta(n-j)\right)_{[n-j]}}{(a n+\beta n)_{[n]}} \\
& \sim \frac{1}{j !} a \theta_{l} \frac{1}{a \theta_{l}+\beta j}\left(a \theta_{l}+\beta j\right)_{[j]} \frac{1}{(a+\beta)^{j}} \phi_{j}^{(n)}, \quad j=0, \ldots, n,
\end{aligned}
$$

where $\phi_{j}^{(n)}$ is the only factor still depending on $n$ and is defined by

$$
\phi_{j}^{(n)}=\frac{\left(a n+\beta n-\left(a \theta_{l}+\beta j-j\right)\right)_{[n]}}{(a n+\beta n)_{[n]}} .
$$

Using Stirling's approximation, we find that

$$
\lim _{n \rightarrow \infty} \phi_{j}^{(n)}=\left(\frac{a+\beta-1}{a+\beta}\right)^{a \theta_{l}+\beta j-j}, \quad j \geq 0 .
$$

Inserting (A.5) into (A.4) then gives

$$
\lim _{n \rightarrow \infty} p_{n-j \mid n}^{(1)}=\frac{a \theta_{l}}{a \theta_{l}+\beta j}\left(\begin{array}{c}
a \theta_{l}+\beta j \\
j
\end{array}\right) \frac{1}{(a+\beta)^{j}}\left(\frac{a+\beta-1}{a+\beta}\right)^{a \theta_{l}+\beta j-j}, \quad j \geq 0,
$$

which is the distribution $g_{\mathcal{N}} \mathcal{B}\left(a \theta_{l} ; \beta,(a+\beta-1) /(a+\beta)\right)$.

\section{Acknowledgements}

We are grateful to the anonymous referee for helpful comments and remarks. The research of M. Gathy was supported by the Belgian FNRS through an FRIA research grant.

\section{References}

Andersson, H. ANd Britton, T. (2000). Stochastic Epidemic Models and Their Statistical Analysis (Lecture Notes Statist. 151). Springer, New York.

Ball, F. (1983). A threshold theorem for the Reed-Frost chain-binomial epidemic. J. Appl. Prob. 20, $153-157$.

Ball, F. and Donnelly, P. (1995). Strong approximations for epidemic models. Stoch. Process. Appl. 55, 1-21.

BALL, F. AND LyNe, O. D. (2001). Stochastic multitype SIR epidemics among a population partitioned into households. Adv. Appl. Prob. 33, 99-123.

Ball, F. AND O'NeILl, P. (1999). The distribution of general final state random variables for stochastic epidemic models. J. Appl. Prob. 36, 473-491.

Ball, F., Mollison, D. and Scalia-Tomba, G. (1997). Epidemics with two levels of mixing. Ann. Appl. Prob. 7, 46-89.

Charnet, R. and Gokhale, D. V. (2004). Statistical inference for damaged Poisson distribution. Commun. Statist. Simul. Comput. 33, 259-269.

Consul, P. C. AND FAMOYe, F. (2006). Lagrangian Probability Distributions. Birkhäuser, Boston, MA.

Daley, D. J. And Gani, J. (1999). Epidemic Modelling: An Introduction. Cambridge University Press.

Di Bucchcianico, A. (1997). Probabilistic and Analytical Aspects of the Umbral Calculus (CWI Tract 119), Amsterdam.

Dobson, I., Carreras, B. A. And Newman, D. E. (2005). A loading-dependent model of probabilistic cascading failure. Prob. Eng. Inf. Sci. 19, 15-32.

Gathy, M. (2007). Distributions Lagrangiennes et de Pólya-Eggenberger généralisée. Working paper, Département de Mathématique, ULB, Bruxelles.

Gontcharoff, W. (1937). Détermination des Fonctions Entières par Interpolation. Hermann, Paris.

Johnson, N. L., Kotz, S. And Kemp, A. W. (1992). Univariate Discrete Distributions, 2nd edn. John Wiley, New York. 
Lefèvre, C. (2006). On the outcome of a cascading failure model. Prob. Eng. Inf. Sci. 20, 413-427.

Lefèvre, C. (2007). First-crossing and ballot-type results for some nonstationary sequences. Adv. Appl. Prob. 39, 492-509.

LEFÈvRe, C. AND PICARD, P. (1990). A nonstandard family of polynomials and the final size distribution of Reed-Frost epidemic processes. Adv. Appl. Prob. 22, 25-48.

Lefèvre, C. And Picard, P. (2005). Nonstationarity and randomization in the Reed-Frost epidemic model. J. Appl. Prob. 42, 950-963.

Lefèvre, C. And Utev, S. (1996). Comparing sums of exchangeable Bernoulli random variables. J. Appl. Prob. 33, $285-310$.

LefÈvre, C. And Utev, S. (1999). Branching approximation for the collective epidemic model. Methodology Comput. Appl. Prob. 1, 211-228.

Lindley, D. V. AND Singpurwalla, N. D. (2002). On exchangeable, causal and cascading failures. Statist. Sci. 2, 209-219.

PicARD, P. AND LefÈvre, C. (1990). A unified analysis of the final size and severity distribution in collective Reed-Frost epidemic processes. Adv. Appl. Prob. 22, 269-294.

PiCARD, P. AND LefÈvre, C. (1991). The dimension of Reed-Frost epidemic models with randomized susceptibility levels. Math. Biosci. 107, 225-233.

PiCARD, P. AND LefÈVRe, C. (1996). First crossing of basic counting processes with lower non-linear boundaries: a unified approach through pseudopolynomials. I. Adv. Appl. Prob. 28, 853-876.

PiCARD, P. AND LEFÈVRE, C. (2003). On the first meeting or crossing of two independent trajectories for some counting processes. Stoch. Process. Appl. 104, 217-242.

Rao, C. R. (1965). On discrete distributions arising out of methods of ascertainment. In Classical and Contagious Discrete Distributions, ed. G. P. Patil, Statistical Publishing Society, Calcutta, pp. 320-332.

Rao, C. R. and Rubin, H. (1964). On a characterization of the Poisson distribution. Sankhyā A 26, $295-298$.

Rota, G.-C., Kahaner, D. And Odlyzko, A. (1973). On the foundations of combinatorial theory. VII. Finite operator calculus. J. Math. Anal. Appl. 42, 684-760.

Shanbhag, D. N. (1977). An extension of the Rao-Rubin characterization of the Poisson distribution. J. Appl. Prob. 14, 640-646.

TAKács, L. (1989). Ballots, queues and random graphs. J. Appl. Prob. 26, 103-112. 